\title{
Identification of Candidate Genes Potentially Relevant to Chamber-Specific Remodeling in Postnatal Ventricular Myocardium
}

\author{
Mario Torrado, Raquel Iglesias, Beatriz Nespereira, and Alexander T. Mikhailov \\ Developmental Biology Unit, Institute of Health Sciences, University of La Coruña, Campus de Oza, As Xubias Street $s / n$, \\ 15006 La Coruña, Spain \\ Correspondence should be addressed to Alexander T. Mikhailov, margot@udc.es
}

Received 8 October 2009; Accepted 7 January 2010

Academic Editor: Aikaterini Kontrogianni-Konstantopoulos

Copyright () 2010 Mario Torrado et al. This is an open access article distributed under the Creative Commons Attribution License, which permits unrestricted use, distribution, and reproduction in any medium, provided the original work is properly cited.

\begin{abstract}
Molecular predisposition of postnatal ventricular myocardium to chamber-dependent (concentric or eccentric) remodeling remains largely elusive. To this end, we compared gene expression in the left (LV) versus right ventricle (RV) in newborn piglets, using a differential display reverse transcription-PCR (DDRT-PCR) technique. Out of more than 5600 DDRT-PCR bands, a total of 153 bands were identified as being differentially displayed. Of these, 96 bands were enriched in the LV, whereas the remaining 57 bands were predominant in the RV. The transcripts, displaying over twofold LV-RV expression differences, were sequenced and identified by BLAST comparison to known mRNA sequences. Among the genes, whose expression was not previously recognized as being chamber-dependent, we identified a small cohort of key regulators of muscle cell growth/proliferation (MAP3K7IP2, MSTN, PHB2, APOBEC3F) and gene expression (PTPLAD1, JMJD1C, CEP290), which may be relevant to the chamber-dependent predisposition of ventricular myocardium to respond differentially to pressure (LV) and volume (RV) overloads after birth. In addition, our data demonstrate chamber-dependent alterations in expression of as yet uncharacterized novel genes, which may also be suitable candidates for association studies in animal models of LV/RV hypertrophy.
\end{abstract}

\section{Introduction}

Ventricular (or cardiac) remodeling is commonly defined as a physiological or pathological process that can occur under various conditions of pressure/volume overload. A common feature of ventricular remodeling is hypertrophy of the cardiomyocytes. The type of cardiac workload determines the pattern of ventricular hypertrophy: volume overload induces eccentric, while pressure overload induces concentric remodeling. Under various pathological conditions, compensatory concentric hypertrophy can lead to eccentric hypertrophy, dilatory ventricular remodelling, and heart failure (reviewed in $[1,2]$ ). The molecular signature of concentric versus eccentric hypertrophy, although poorly defined as yet, is nevertheless of critical relevance in cardiac basic and clinical research [3-8].

The early neonatal heart is a conventional model for the study of distinct patterns of ventricular hypertrophy (i.e., concentric versus eccentric). At birth, cardiomyocytes begin to enlarge in response to the demands of physiological workload, as opposed to processes driven predominantly by developmental mechanisms. Particularly, the left ventricle (LV) is exposed to a higher-pressure overload in comparison to the right ventricle (RV), which is exposed to a relatively higher-volume overload. As a result, the LV undergoes rapid concentric hypertrophy, while the RV undergoes eccentric hypertrophy associated with dilatory RV-chamber remodeling. Our previous data revealed differences in the expression of cardiac ankyrin repeat domain 1 factor (ANKRD1/CARP) between the LV and RV before the appearance of morphologically identifiable signs of LV-concentric or RV-eccentric hypertrophy in newborn piglets [9]. Other research reported certain LV/RV-specific metabolic differences in normal and ischemic newborn piglet heart [7]. We interpreted these results as reflecting a certain type of molecular predisposition of newborn ventricular myocardium to LV-concentric and $\mathrm{RV}$-eccentric remodeling during postnatal development. 


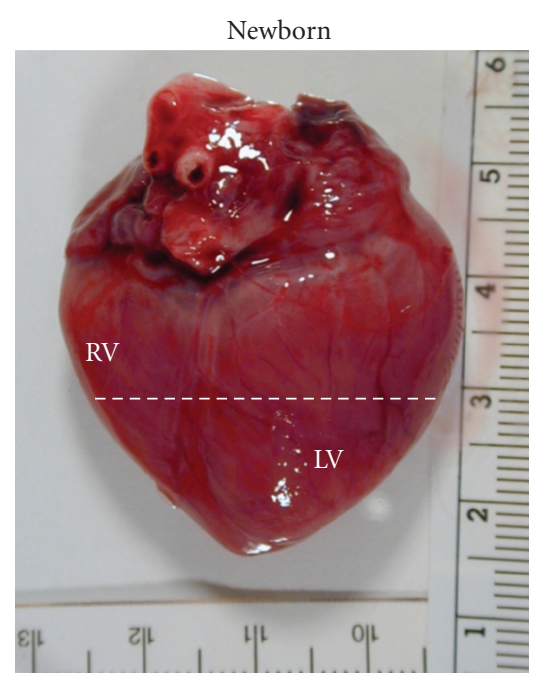

(a)

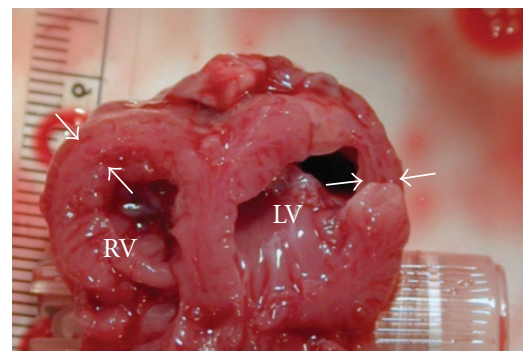

(c)

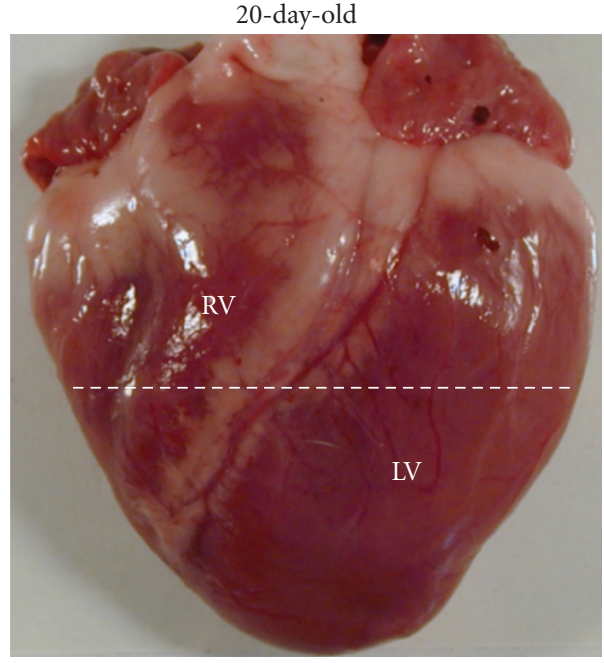

(b)

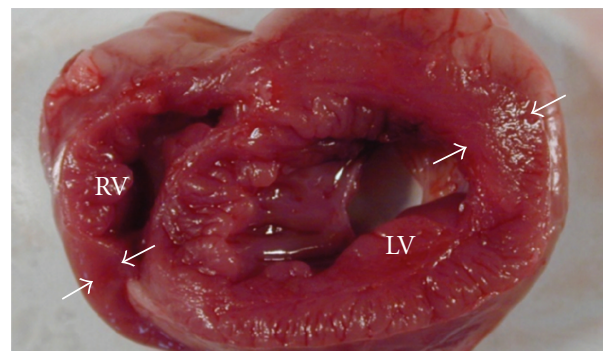

(d)

Figure 1: Heart dimensions ( $\mathrm{a}$ and $\mathrm{b}$ ) and left/right ventricular cross-sections (c and d) of newborn and 20-day-old piglets. LV/RVleft/right ventricle. (a), (b) Levels of cross sections are shown by dotted lines. (c), (d) Boundaries of the LV/RV free wall are marked by white arrows.

In the present study, we focused on large-scale transcriptomic analysis to compare differences in gene expression levels in the LV versus RV in newborn piglets. Given that commercially available DNA microarray platforms suitable for performing transcriptional profiling in pig are still poorly developed, we conducted comparative LV versus RV gene expression profiling in newborn piglets using mRNA differential display (DDRT-PCR). In addition, unlike microarraybased platforms, DDRT-PCR can be used to detect expression changes in both known and novel transcripts including alternate splice variants [10]. This approach allowed us (1) to perform an unbiased assessment of genes which expression is predominantly associated with piglet LV or RV myocardium and (2) to distil a large body of expression data into a discrete set of candidate genes for which regulation was not previously recognized as being chamber-dependent. Further studies on these differentially regulated genes will likely lead to the identification of additional novel gene families and pathways involved in the chamber-dependent response of ventricular myocardium to a variety of physiological and pathological stimuli.

\section{Materials and Methods}

2.1. Animals and Tissue Sampling. Animals were treated and cared for in accordance with the European commission directive 86/609/EEC on the protection of animals used for experimental and other scientific purposes, and all animal protocols were approved by the ethical research committee of Galicia (Spain). Newborn (10-12 hours after birth) Large White piglets were obtained from a local commercial breeder (La Coruña, Galicia) and maintained in an automatic nursery system (Nütinger System). The newborn and 20day-old animals were anaesthetized, the thoracic cavity was opened through a median sternotomy, and the entire heart was rapidly removed, weighed, and photographed while still beating. Then the isolated heart was placed on an ice-cold petri dish, partially sectioned at the midpoint of the LV length and photographs of the open ventricular chambers were taken (Figure 1). Immediately after this step, the LV and RV free walls were dissected, flash frozen in liquid nitrogen, and stored at $-80^{\circ} \mathrm{C}$ until study.

2.2. RNA Isolation. Deep-frozen tissue samples (100$150 \mathrm{mg}$ ), encompassing the full thickness of the free wall of the LV and RV ventricle, were directly disrupted in RLT buffer (Qiagen) using a high-speed rotor-stator homogenizer (Ultra-Turrax T8, Germany), digested with Proteinase K (Qiagen), loaded onto a RNeasy Midi column (Qiagen), subjected to on-column digestion of DNA with RNase-free DNase (Qiagen) and the analysis proceeded in accordance 
with the manufacturer's recommendations. Resulting RNA preparations were ethanol-precipitated, resolved in RNasefree water, and kept at $-80^{\circ} \mathrm{C}$. RNA yield and purity was determined spectrophotometrically at $260-280 \mathrm{~nm}$ and RNA integrity was verified by running samples on $1.5 \%$ agarose gels and staining with ethidium bromide.

2.3. Differential Display mRNA Analysis. The reverse transcription-PCR differential display (DDRT-PCR) analysis was performed as described [11] with minor modifications [12]. To yield starting material for the DDRT-PCR, total RNA preparations independently isolated from the LV and $\mathrm{RV}$ of three newborn piglets were, respectively, pooled at equal ratios, and $4 \mu \mathrm{g}$ of RNA was reverse transcribed using the SuperScript III (Invitrogen) and T7-oligo-dT primer. Pooled first-strand cDNAs were amplified side-by-side by PCR using 230 different primer combinations (10 two-baseanchored oligo-dT and 23 arbitrary primers purified by HPLC, Table 1).

Nontemplate (NT) and non-RT RNA (N-RT) template reactions were used as negative controls. In each DDRTPCR set-up, reactions were performed at least in duplicate to test whether differences in LV/RV gene expression are likely to be real. PCR was performed, using the AmpliTaq DNA polymerase (Invitrogen), under the following conditions: initial denaturation $\left(94^{\circ} \mathrm{C}, 2\right.$ minutes), stage I (5 cycles, each of which included: $94^{\circ} \mathrm{C}, 30$ seconds; $40^{\circ} \mathrm{C}, 1$ minute; $72^{\circ} \mathrm{C}, 1$ minute), stage II ( 25 cycles, each on which included: $94^{\circ} \mathrm{C}, 30$ seconds; $50^{\circ} \mathrm{C}, 1$ minute; $72^{\circ} \mathrm{C}, 1$ minute), and final extension $\left(72^{\circ} \mathrm{C}, 10\right.$ minutes), sample store at $6^{\circ} \mathrm{C}$. PCR-amplified products were subjected to fractionation on $8 \%$ polyacrylamide gels (PAAG) (Mini-Protean-III, BioRad) and fluorescently stained by SYBR Green I (Sigma). Image acquisition and intensity of bands were estimated by densitometry (VersaDoc 1000) and Quantity One software (Bio-Rad). Differentially regulated amplification products were defined as those bands that were similarly displayed at least in two experimental replicates. Using a sharp, sterile razor blade, a rectangular piece of gel corresponding to an individual band of interest on the PAAG was excised and electroeluted (D-tube Electroelution Kit, Novagen). After a short centrifugation, the eluate was transferred to a clean tube. The extracted DNA was used directly as the template for PCR with T7 and M13 reamplification primers (see Table 1). Cycling conditions were as described for DDRT-PCR except stage I at $45^{\circ} \mathrm{C}$ and stage II $(20$ cycles $)$ at $55^{\circ} \mathrm{C}$. After reamplification, each PCR reaction was electrophoresed through a $1.5 \%$ agarose gel with ethidium bromide to assure that the correct sized fragment was amplified. Reamplified cDNA fragments were eluted (QIAquick Gel Extraction Kit, Qiagen), cloned into pCRII-TOPO vector (Invitrogen), and sequenced by (Secugen), (Madrid, Spain). The nucleotide sequences obtained were compared with known sequences by searching the GenBank database with BLAST algorithms.

2.4. Quantitative RT-PCR. Differential gene expression was further confirmed by real-time quantitative PCR (qRT-PCR) as described [13] using Bio-Rad IQ5 instrument and Bio-Rad
TABLE 1: Primers used in differential display RT-PCR analysis.

\begin{tabular}{|c|c|}
\hline & T7-Oligo(dT) \\
\hline & ACGACTCACTATAGGGCTTTTTTTTTTTTTT \\
\hline \multicolumn{2}{|c|}{ two-base anchored oligo-dT antisense primers* } \\
\hline H01 & ACGACTCACTATAGGGCTTTTTTTTTTTTGA \\
\hline H02 & ACGACTCACTATAGGGCTTTTTTTTTTTTGC \\
\hline H03 & ACGACTCACTATAGGGCTTTTTTTTTTTTGG \\
\hline H04 & ACGACTCACTATAGGGCTTTTTTTTTTTTGT \\
\hline H05 & ACGACTCACTATAGGGCTTTTTTTTTTTTCA \\
\hline H06 & ACGACTCACTATAGGGCTTTTTTTTTTTTCC \\
\hline H07 & ACGACTCACTATAGGGCTTTTTTTTTTTTCG \\
\hline H08 & ACGACTCACTATAGGGCTTTTTTTTTTTTAA \\
\hline H09 & ACGACTCACTATAGGGCTTTTTTTTTTTTAC \\
\hline $\mathrm{H} 10$ & ACGACTCACTATAGGGCTTTTTTTTTTTTAG \\
\hline \multicolumn{2}{|r|}{ 10-mer arbitrary sense primers** } \\
\hline A01 & ACAATTTCACACAGGACGACTCCAAG \\
\hline A02 & ACAATTTCACACAGGAGCTAGCATGG \\
\hline A03 & ACAATTTCACACAGGAGACCATTGCA \\
\hline A04 & ACAATTTCACACAGGAGCTAGCAGAC \\
\hline A05 & ACAATTTCACACAGGAATGGTCGTCT \\
\hline A06 & ACAATTTCACACAGGATACAACGAGG \\
\hline A07 & ACAATTTCACACAGGATGGATTGGTC \\
\hline A08 & ACAATTTCACACAGGATGGTAAAGGG \\
\hline A09 & ACAATTTCACACAGGATAAGCCTAGC \\
\hline A10 & ACAATTTCACACAGGAGATCTCAGAC \\
\hline A11 & ACAATTTCACACAGGAACGCTAGTGT \\
\hline A12 & ACAATTTCACACAGGAGGTACTAAGG \\
\hline A13 & ACAATTTCACACAGGAGTTGCACCAT \\
\hline A14 & ACAATTTCACACAGGATCCATGACTC \\
\hline A15 & ACAATTTCACACAGGACTTTCTACCC \\
\hline A16 & ACAATTTCACACAGGATCGGTCATAG \\
\hline A17 & ACAATTTCACACAGGACTGCTAGGTA \\
\hline A18 & ACAATTTCACACAGGATGATGCTACC \\
\hline A19 & ACAATTTCACACAGGATTTTGGCTCC \\
\hline A20 & ACAATTTCACACAGGATCGATACAGG \\
\hline A21 & ACAATTTCACACAGGACAGGCAGCAG \\
\hline A23 & ACAATTTCACACAGGATATGGCGCCG \\
\hline A24 & ACAATTTCACACAGGAGCTGAACCGG \\
\hline \multicolumn{2}{|r|}{ primers for reamplification of DD bands } \\
\hline T7 & GTAATACGACTCACTATAGGGC \\
\hline M13rev-48p & AGCGGATAACAATTTCACACAGGA \\
\hline
\end{tabular}

*Each anchor primer has T7 sequence (bold) on the $5^{\prime}$ end.

**Each arbitrary primer has M13 sequence (bold) on the $5^{\prime}$ end.

SYBR Green Mix [14, 15]. Whenever possible, the primer pairs were designed to be located in different exons of a given sequence. Individual heart-matched LV/RV cDNAs isolated from three newborn and three 20-day-old piglets were used as templates. Each primer pair used yielded a single peak of dissociation on the melting curve and a single band with expected size on PAAG [12]. A negative NT and NRT controls were included in each reaction set. Detection of ribosomal protein L19 (RPL19) mRNA was used to normalize the expression of target mRNAs. The efficiency of 


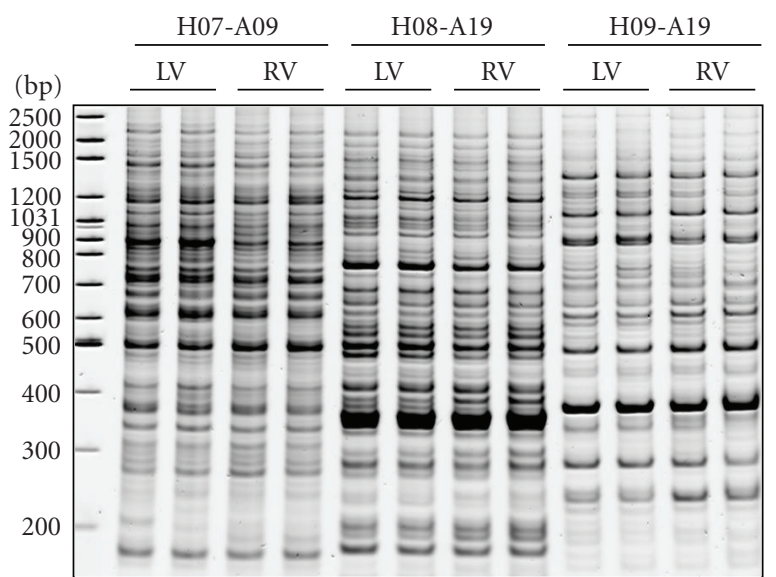

(a)

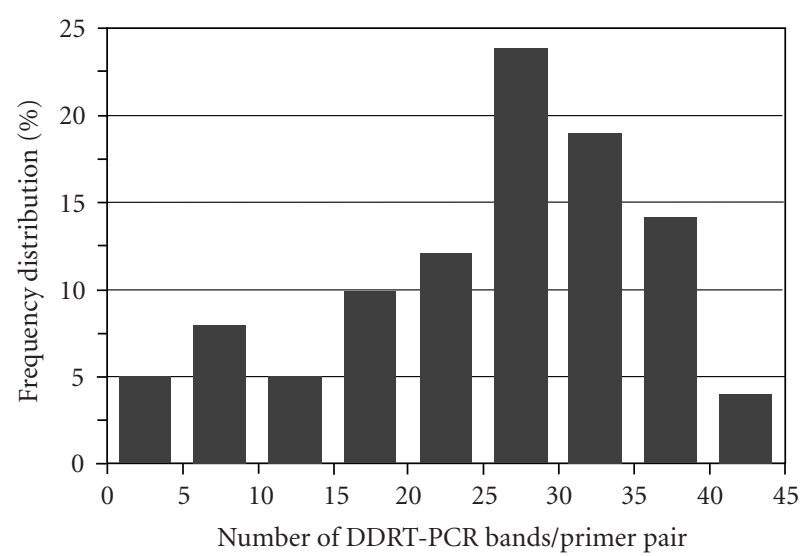

(b)

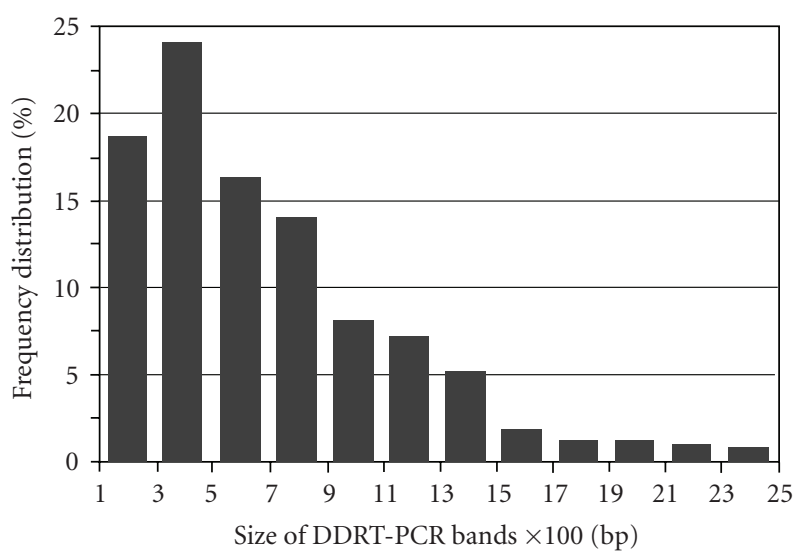

(c)

FIGURE 2: Differential display (DDRT-PCR) analysis of gene expression in left/right ventricles (LV/RV) of newborn piglets. (a) Representative gel images of DDRT-PCR bands amplified with three distinct sets of primer combinations (H07-A09, H08-A19, and H09-A19), showing highly reproducible band patterns in each replicate. Nondenaturing 8\% PAAG poststained with SYBR Green I. 200-2500 bp-DNA size standards (GeneRuler DNA ladder mix, Fermentas). (b and c) Number and size distribution frequencies of bands generated by DDRT-PCR.

target and reference amplification was tested and found to be approximately equal. Results were defined as the target genes expression normalized against rpl19 gene expression in both ventricles. Fold changes were calculated using the $\mathrm{C}_{T}$ method. Primer sequences and additional details on $\mathrm{qRT}$ PCR are available upon request.

2.5. Data Analysis. Values were expressed as means \pm SEM. mRNA expression was quantified using the comparative threshold cycle method. Statistical analyses were performed with the SPSS 13 software. A $P$ value $<.05$ was considered to be statistically significant.

\section{Results}

3.1. DDRT-PCR Analysis Allows Reliable Transcriptomic Profiling of Ventricular Myocardium in Newborn Piglets. For mammalian cells, it was calculated that 20 arbitrary in conjunction with 12 anchored primers would statistically amplify all mRNA sequences [16]. We used 23 arbitrary and
10 two-base-oligo(dT) anchored primers (Table 1), resulting in 230 display primer combinations. A total of about 5,600 distinct cDNA fragments corresponding to genes expressed in piglet $\mathrm{LV} / \mathrm{RV}$ myocardium were detected. A representative example of DDRT-PCR banding patterns is illustrated in Figure 2(a).

The average number of bands generated by one primer pair was 26 , the minimum was 0 , and the maximum was 44. About $70 \%$ of the primer pairs produced $20-40$ bands (Figure 2(b)). Size distribution analysis of cDNA bands generated by DDRT-PCR revealed a minor fraction of shortsized (100-300 nt) bands, while the fragments with a size from 300 to $1,000 \mathrm{nt}$, which is a preferable choice for cloning and sequencing, made up about $60 \%$ of all detected bands (Figure 2(c)).

Taken together, the results indicated that under our experimental conditions, transcript-banding patterns generated by DDRT-PCR could be sufficient for comparative expression analysis of the LV versus RV myocardium of newborn piglets. 


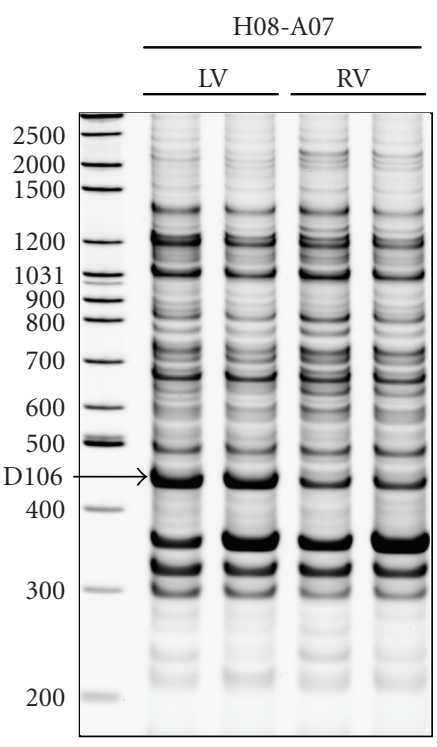

(a)

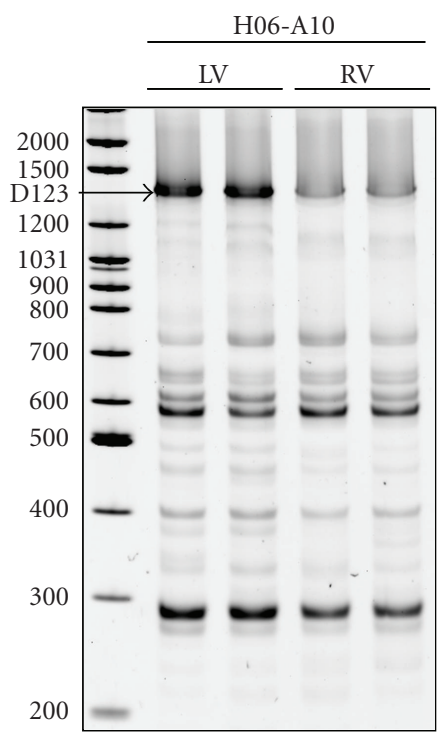

(c)

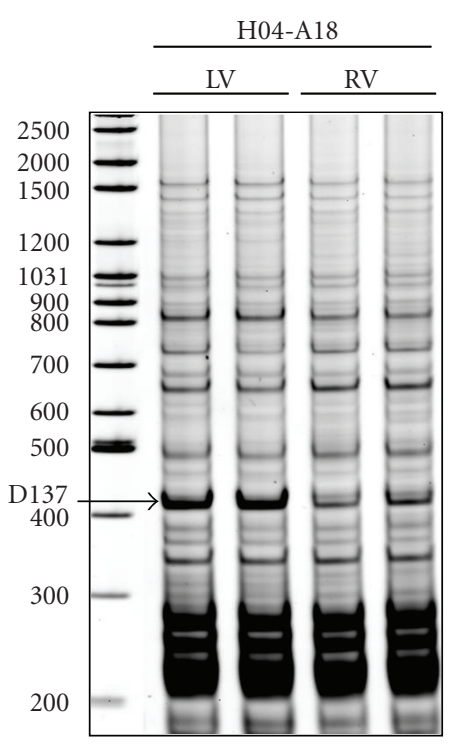

(b)

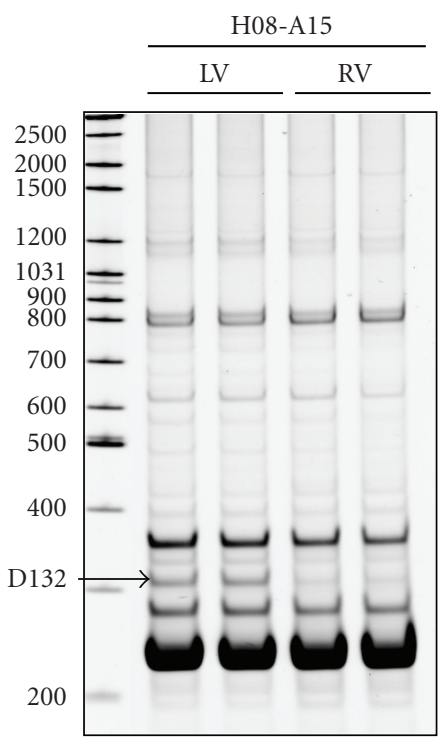

(d)

FIGURE 3: Examples of the bands, displaying over twofold LV versus RV expression differences in newborn piglets. The primer pairs used for DDRT-PCR amplifications are shown. Nondenaturing 8\% PAAG poststained with SYBR Green I. LV/RV: left/right ventricle. 200-2500 bp: DNA size standards (GeneRuler DNA ladder mix, Fermentas). Arrows: the bands (D106, D137, D123, and D132), which correspond to the transcripts differentially displayed between LV and RV. For further details see Table 2.

\subsection{DDRT-PCR Profiling Identifies Differentially Expressed} Genes in the LV versus RV Myocardium of Newborn Piglets. Direct side-by-side comparison of the mRNAs between the $\mathrm{LV}$ and RV of the newborn piglet heart revealed that the majority of profiled genes (97\%) were similarly expressed in both ventricles. Out of more than 5,600 DDRT-PCR bands amplified by the primer combinations used, a total of 153 bands, ranging in size from 300 to $1,000 \mathrm{nt}$, were identified as being qualitatively differentially displayed. Of these, 96 transcripts were enriched in the $\mathrm{LV}$, whereas the remaining 57 were predominant in the RV.
Figure 3 illustrates the relative differential expression of a representative set of bands in the LV as compared to the RV myocardium. Once differentially displayed PCR products were detected, the fragments which displayed over twofold LV-RV expression differences (40 bands) were recovered from gels, reamplified, cloned, and sequenced. The differential expression of these genes was further confirmed using qRT-PCR analysis. In this manner, over $80 \%$ (32 bands) of the selected bands were confirmed to be differentially expressed in the two ventricular chambers of newborn piglets (Table 2). 


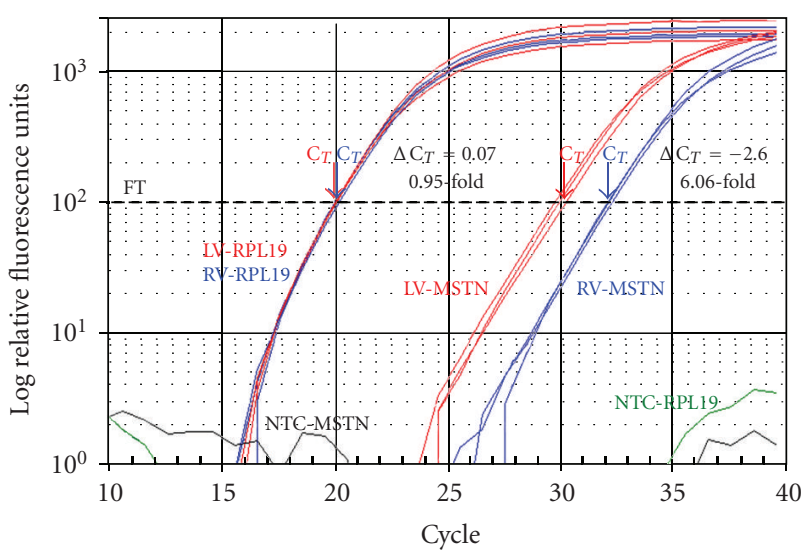

(a)

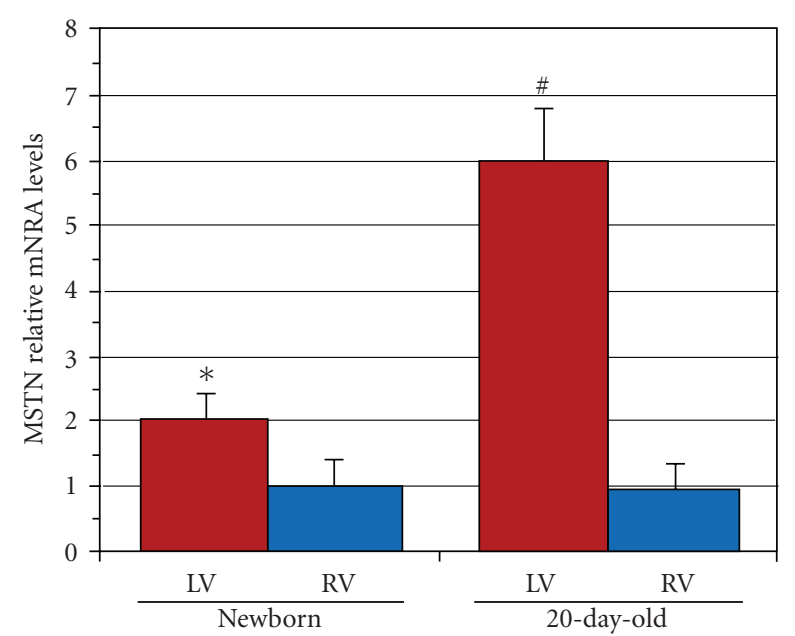

(b)

FIGURE 4: Estimation of myostatin (MSTN) mRNA levels in the LV and RV of newborn and 20-day-old piglets. (a) Representative qPCR amplification plot of MSTN mRNA levels in the LV (red) and RV (blue) of three 20-day-old piglet hearts. Internal RPL19 reference levels in the LV (red) and RV (blue) are shown. Arrows: threshold cycle $\left(\mathrm{C}_{T}\right)$. FT: fluorescent threshold. $\Delta \mathrm{C}_{T}$ : differences in threshold cycles for target and reference. NTC: nontemplate controls. B: MSTN mRNA levels in the LV versus RV ventricle of newborn and 20-day-old piglets. ${ }^{*} P<.05$, newborn piglets $(n=3)$. ${ }^{\#} P<.05,20$-day-old piglets $(n=3)$.

The BLAST searches for sequence similarity revealed that 6 of the 32 cloned cDNA fragments with confirmed differential expression are potentially novel transcripts with no significant match in the current databases, suggesting that they may either encode as yet uncharacterized proteins or correspond to unknown regions of identified genes (untranslated, nonconserved regions). The remaining $26 \mathrm{cDNA}$ sequences were identified by BLAST sequence comparisons as genes related to modulation of gene expression (PTPLAD1, PHB2, CEP290, JMJD1C), regulation of cell growth and differentiation (MSTN, MAP3K71P2, APOBEC3F, PHB2), biomechanical stress sensing and myofibrilar assembly (TTN, ANKRD1), muscle contraction (TNNT2, ACTC1), extracellular matrix remodeling (ADAMTS3, COL1A2), calcium control (SLC8A1), and energy metabolism (ATP5C1, ND6).

Table 2 provides details of the extent of relative LV/RV upregulation (fold change) as well as the known function(s) of identified genes. Among the differentially expressed genes, only a small portion displayed over 4-fold expression differences between LV and RV (PTPLAD1, TPM2, ACTC1, ANKRD1, ANKRD1-I8, PDE3A, D162, TNMD, D170). In this sense, chamber-dependent regulation of expression of these known and novel transcripts may be primarily associated with different patterns of postnatal ventricular remodeling.

MSTN (myostatin) characterized by LV-predominant expression in newborn myocardium also stood out as an interesting candidate, given its roles in cell growth and proliferation. Recently, it has been demonstrated that MSTN is a potent repressor of cardiac muscle cell proliferation and growth, and that in vivo loss of MSTN induces eccentric hypertrophy associated with enhanced responsiveness of ventricular myocytes to beta-adrenergic stimulation [17, $18]$. We, therefore, examined this gene expression in both ventricles at advanced stages of postnatal development when morphological differences between concentric (LV) and eccentric (RV) remodeling become evident, that is in 20day-old piglets (see Figure 1(d)). The LV/RV MSTN mRNA ratio found in newborn piglets (i.e., 2 : 1) was significantly amplified in 20-day-old animals (i.e., $6: 1$ ) due to MSTN upregulation in the LV of the latter age group, while the gene's expression levels in the RV were similar in two groups studied (Figure 4). The results indicate that in neonatal piglets a process of RV-eccentric remodeling is associated with the same relative low MSTN level as was found in the $\mathrm{RV}$ at birth.

Collectively, the comparison of gene expression between the LV and RV shortly after birth, when LV/RV loading conditions are dramatically changed as compared to the latefetal period, demonstrates that such analysis provides clues for identifying hallmark genes whose expression is regulated in a chamber-dependent manner at the earliest stages of postnatal LV-concentric and RV-eccentric remodeling.

\section{Discussion}

The DDRT-PCR technique, which was first developed in 1992 [19], is still the method of choice for an unbiased comparison of mRNA expression patterns between samples that are very similar and often results in identification of nonabundant, rare, or novel transcripts [10, 20, 21].

Using a nonradioactive DDRT-PCR technique, we identified the transcripts that reproducibly showed different expression levels between LV and RV in newborn piglets. These differences do not correlate with either cardiomyocyte cell volume [22] or ventricular wall thickness ([9]; see also Figure 1(b), this work), which are practically equal in both piglet ventricles during or shortly after birth. Thus, in 


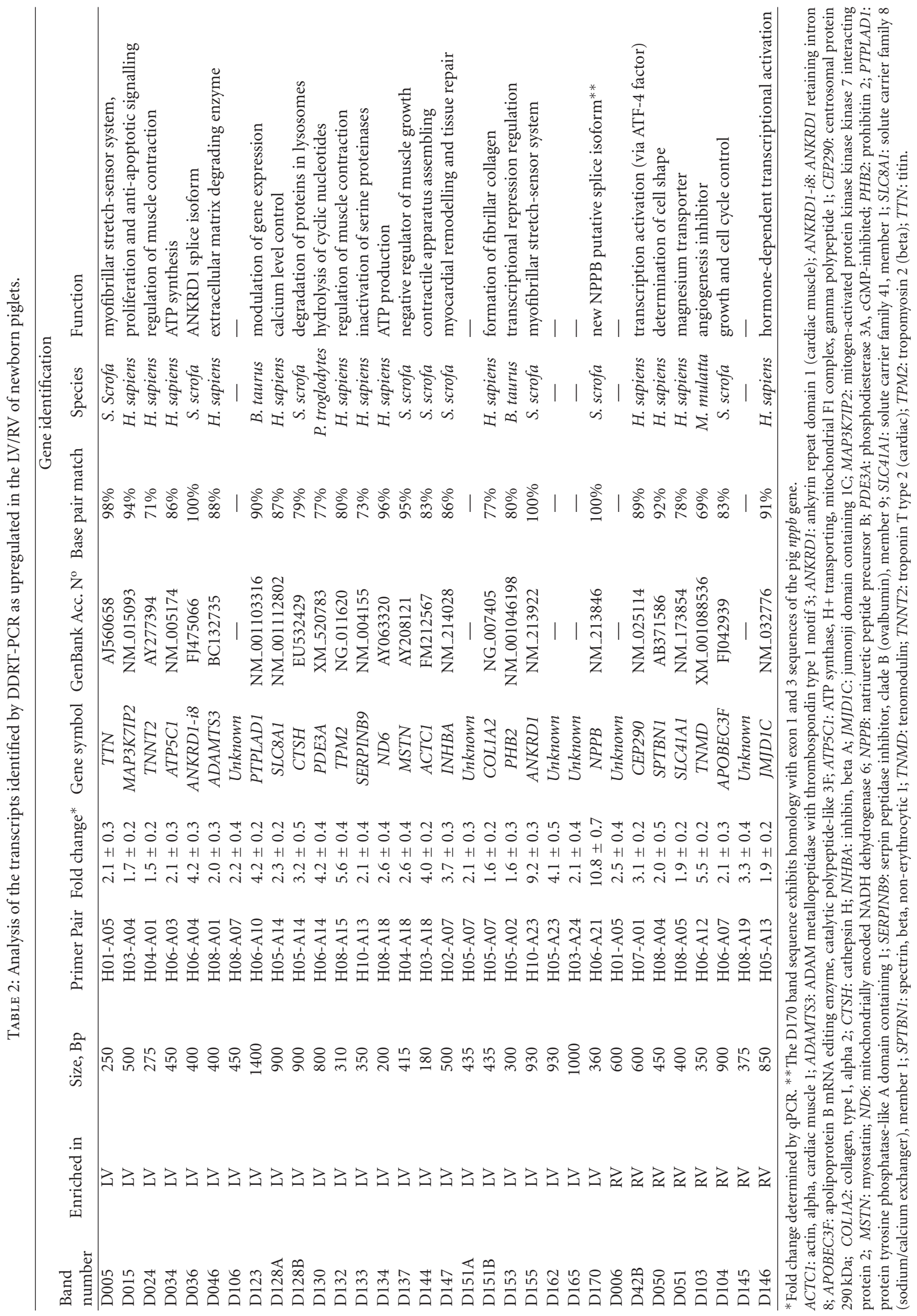


this system a molecular prepattern precedes the appearance of morphologically identifiable signs of LV-concentric and RV-eccentric hypertrophy. We suggest that the observed differences in gene expression are intrinsic to the distinct molecular makeup of the LV versus RV rather than to their hyperplastic/hypertrophic growth status, which is similar in both ventricles at birth. Further, the content of certain wellknown markers of cardiomyocyte hypertrophy (beta-myosin heavy chain and myosin light chain 2 ventricular) was found to be similar in both the LV and RV of newborn piglets [9]. Moreover, expression levels of the transcriptional cofactor, myocardin, which induces cardiomyocyte hypertrophy [23, 24 ], are equal in both ventricles of these animals [25]. Therefore, it seems reasonable to interpret the differences in gene expression detected in our present work as indicative of an L-R molecular predisposition of the newborn myocardium to respond to dramatic changes of the hemodynamic loads shortly after birth when the LV is exposed to a higherpressure load (concentric hypertrophy promoting condition) in comparison to the RV, which is exposed to a highervolume load (eccentric hypertrophy promoting condition).

The vast majority of the transcripts differentially expressed in the LV and RV of newborn piglets correspond to genes which were not previously known to be asymmetrically expressed in the LV versus RV myocardium, excepting those coding for beta-spectrin [4], ANKRD1 [9], BNP $[6,9]$, calcium ATPase, matrix metalloproteinases, type 1 procollagens, and troponins [3]. In addition, other reports demonstrated that transcripts for proteins such as fibronectin, alpha-myosin heavy chain and transforming growth factor [26], and cytochrome c oxidase and heart isoforms of uncoupling proteins [27] are asymmetrically enriched in the LV versus RV mammalian myocardium.

Regulatory mechanisms resulting in LV/RV transcriptional differences in the newborn and early neonatal heart are largely unknown, but of special interest, because the functionally different roles of the two ventricles become apparent after birth. Our study characterizes the transcription status of the LV and RV at birth rather than the establishment of LV/RV transcription differences in the course of development [28]. In embryonic and fetal heart, expression of a number of transcription factors, including Hand1, Hand2, and Tbx5, shows LV/RV differences [29, 30]. We found [9] that Hand1 and Hand 2 are equally expressed in both the LV and RV of newborn piglets, suggesting that these factors are not involved in maintaining $L / R$ ventricular transcriptional differences after birth.

In this work, among the genes whose expression levels differentiate between the LV and RV, there is a small cohort of genes which could be involved in concentric versus eccentric hypertrophy signalling (see Table 2). In this regard, several key regulators of muscle cell growth and proliferation (MAP3K7IP2, MSTN, PHB2, APOBEC3F) and gene expression (PTPLAD1, JMJD1C, CEP290) are differentially expressed between LV and RV piglet myocardium that may be relevant to intrinsic differences [31] that can regulate the chamber-dependent response of ventricular myocardium to workload. Interestingly, transition from "early" to "late" hypertension-induced hypertrophy in young adult rats is associated with predominant changes in expression of cell growth/proliferation and signal transduction factors [32].

Sequence analysis of the 32 cDNAs chosen based on differential LV/RV screening revealed a number of sequences, which may correspond to either previously uncharacterized genes or yet unidentified splice variants of the known cardioexpressed genes. In this sense, identification of the D36 fragment sequence (see Table 2) as being completely identical to that located within intron 8 of the pig ankrd1 gene led us to isolate and characterize three novel alternatively spliced ankrd1 variants which are predominantly expressed in the LV of neonatal and adult pig and human hearts and markedly upregulated in the ventricular myocardium at experimental heart failure [12]. Similarly, the D170 fragment (see Table 2), exhibiting homology with exon 1 and 3 sequences of the pig $n p p b$ gene, may represent a new form of alternative splicing of this cardioprotective factor.

Various cardiac disease states can result in an imbalance of chamber-associated expression patterns in ventricular myocardium. In the rat infarct model, a shift in chamberdependent gene expression towards relative downregulation of gene expression in the RV as compared to the LV has been reported [3]. In the porcine model of cardiotoxic cardiomyopathy we have demonstrated that the normal asymmetric LV/RV pattern of ANKRD1 mRNA and protein distribution was completely abolished at end-stage heart failure; improvement of cardiac performance resulted in the restoration of this gene's LV/RV asymmetric expression [9]. In the pig model of volume overload (eccentric hypertrophy promoting condition), angiotensinogen and preproendothelin expression levels were significantly upregulated in the RV while remaining uncharged in the LV [31]. In the mouse model of RV pressure-overload hypertrophy, over 10 transcripts showed significant upregulation in the afterload stressed RV, but not in the afterload stressed LV, including three genes from the Wnt signaling pathway, and genes involved in apoptosis [33]. In young rats, chronic hypoxia resulted in a shift from an $\mathrm{LV}$ - to an $\mathrm{RV}$-predominant pattern in cytochrome c oxidase expression [27].

In sum, although not all of the identified genes with differential LV/RV expression have a clearly defined cardiacrelated function(s) at this time, the results of our work do advance the understanding of the complex mechanisms that could be involved in concentric versus eccentric remodeling of ventricular myocardium under normal conditions. More broadly, the identification of specific expression signatures of concentric versus eccentric hypertrophy may be useful in the elucidation of molecular pathways involved not only in physiological but also in pathological myocardial remodelling and heart failure.

\section{Conclusions}

Using an unbiased DDRT-PCR analysis, we were able to identify a set of genes with divergent LV versus RV expression. To our knowledge, this is the first study to account for large-scale gene expression profiling in early neonatal myocardium in mammals which revealed a certain molecular predisposition of the LV and RV, respectively, to concentric 
or eccentric hypertrophic remodeling. The reliability of these findings is supported by confirmation of the results by qRT-PCR and recognition of a fraction of the differentially expressed genes as known genes involved in pathological ventricular remodeling and heart failure. In addition, our data demonstrate chamber-dependent alterations in the expression of as yet uncharacterised novel genes that may be associated with different patterns of ventricular hypertrophic remodeling and can be used to study a board range of heart disease phenotypes.

\section{Acknowledgments}

HPLC-purified primers for DDRT-PCR were a generous gift from Dr. Max Rothschild (Iowa State University, USA). This work was partially supported by Grants (SAF2004-01462 and SAF2008-00337) from the Spanish Ministry of Science and Innovation and by a Grant (08CSA008161PR) from the Autonomic Government of Galicia.

\section{References}

[1] J. Heineke and J. D. Molkentin, "Regulation of cardiac hypertrophy by intracellular signalling pathways," Nature Reviews Molecular Cell Biology, vol. 7, no. 8, pp. 589-600, 2006.

[2] D. Hilfiker-Kleiner, U. Landmesser, and H. Drexler, "Molecular mechanisms in heart failure. Focus on cardiac hypertrophy, inflammation, angiogenesis, and apoptosis," Journal of the American College of Cardiology, vol. 48, no. 9, pp. A56-A66, 2006.

[3] S. S. Chugh, S. Whitesel, M. Turner, C. T. Roberts Jr., and S. R. Nagalla, "Genetic basis for chamber-specific ventricular phenotypes in the rat infarct model," Cardiovascular Research, vol. 57, no. 2, pp. 477-485, 2003.

[4] R. Tabibiazar, R. A. Wagner, A. Liao, and T. Quertermous, "Transcriptional profiling of the heart reveals chamberspecific gene expression patterns," Circulation Research, vol. 93, no. 12, pp. 1193-1201, 2003.

[5] K. Lemmens, V. F. M. Segers, M. Demolder, M. Michiels, P. Van Cauwelaert, and G. W. De Keulenaer, "Endogenous inhibitors of hypertrophy in concentric versus eccentric hypertrophy," European Journal of Heart Failure, vol. 9, no. 4, pp. 352-356, 2007.

[6] B. D. Kaufman, M. Desai, S. Reddy, et al., "Genomic profiling of left and right ventricular hypertrophy in congenital heart disease," Journal of Cardiac Failure, vol. 14, no. 9, pp. 760-767, 2008.

[7] D. Quaglietta, M. P. Belanger, and C. Wittnich, "Ventriclespecific metabolic differences in the newborn piglet myocardium in vivo and during arrested global ischemia," Pediatric Research, vol. 63, no. 1, pp. 15-19, 2008.

[8] S. Champetier, A. Bojmehrani, J. Beaudoin, et al., "Gene profiling of left ventricle eccentric hypertrophy in aortic regurgitation in rats: rationale for targeting the $\beta$-adrenergic and renin-angiotensin systems," American Journal of PhysiologyHeart and Circulatory Physiology, vol. 296, no. 3, pp. H669H677, 2009.

[9] M. Torrado, E. Lopez, A. Centeno, A. Castro-Beiras, and A. T. Mikhailov, "Left-right asymmetric ventricular expression of CARP in the piglet heart: regional response to experimental heart failure," European Journal of Heart Failure, vol. 6, no. 2, pp. 161-172, 2004.
[10] M. Steinau and M. S. Rajeevan, "RNA profiling in peripheral blood cells by fluorescent differential display PCR," Methods in Molecular Biology, vol. 496, pp. 211-222, 2009.

[11] K. Kokame, H. Kato, and T. Miyata, "Nonradioactive differential display cloning of genes induced by homocysteine in vascular endothelial cells," Methods, vol. 16, no. 4, pp. 434443, 1998.

[12] M. Torrado, R. Iglesias, B. Nespereira, A. Centeno, E. Lopez, and A. T. Mikhailov, "Intron retention generates ANKRD1 splice variants that are co-regulated with the main transcript in normal and failing myocardium," Gene, vol. 440, no. 1-2, pp. 28-41, 2009.

[13] M. S. Rajeevan, D. G. Ranamukhaarachchi, S. D. Vernon, and E. R. Unger, "Use of real-time quantitative PCR to validate the results of cDNA array and differential display PCR technologies," Methods, vol. 25, no. 4, pp. 443-451, 2001.

[14] M. Torrado, B. Nespereira, Y. Bouzamayor, A. Centeno, E. Lopez, and A. T. Mikhailov, "Differential atrial versus ventricular ANKRD1 gene expression is oppositely regulated at diastolic heart failure," FEBS Letters, vol. 580, no. 17, pp. 4182-4187, 2006.

[15] M. Torrado, A. Centeno, E. López, and A. T. Mikhailov, "In vivo forced expression of myocardin in ventricular myocardium transiently impairs systolic performance in early neonatal pig heart," International Journal of Developmental Biology, vol. 53, no. 8-10, pp. 1457-1467, 2009.

[16] S. Yang and P. Liang, "Global analysis of gene expression by differential display: a mathematical model," Methods in Molecular Biology, vol. 317, pp. 3-21, 2006.

[17] B. D. Rodgers, J. P. Interlichia, D. K. Garikipati, et al., "Myostatin represses physiological hypertrophy of the heart and excitation-contraction couping," The Journal of Physiology, vol. 587, pp. 4873-4886, 2009.

[18] T. E. Callis, K. Pandya, H. Y. Seok, et al., "MicroRNA-208a is a regulator of cardiac hypertrophy and conduction in mice," Journal of Clinical Investigation, vol. 119, no. 9, pp. 2772-2786, 2009.

[19] P. Liang and A. B. Pardee, "Differential display of eukaryotic messenger RNA by means of the polymerase chain reaction," Science, vol. 257, no. 5072, pp. 967-971, 1992.

[20] M. M. Shen, "Identification of differentially expressed genes in mouse development using differential display and in situ hybridization," Methods, vol. 24, no. 1, pp. 15-27, 2001.

[21] D. Tesfaye, S. Ponsuksili, K. Wimmers, M. Gilles, and K. Schellander, "Identification and quantification of differentially expressed transcripts in in vitro-produced bovine preimplantation stage embryos," Molecular Reproduction and Development, vol. 66, no. 2, pp. 105-114, 2003.

[22] C. T. Beinlich, C. J. Rissinger, and H. E. Morgan, "Mechanisms of rapid growth in the neonatal pig heart," Journal of Molecular and Cellular Cardiology, vol. 27, no. 1, pp. 273-281, 1995.

[23] C. Badorff, F. H. Seeger, A. M. Zeiher, and S. Dimmeler, "Glycogen synthase kinase $3 \beta$ inhibits myocardin-dependent transcription and hypertrophy induction through site-specific phosphorylation," Circulation Research, vol. 97, no. 7, pp. 645654, 2005.

[24] W. Xing, T.-C. Zhang, D. Cao, et al., "Myocardin induces cardiomyocyte hypertrophy," Circulation Research, vol. 98, no. 8, pp. 1089-1097, 2006.

[25] M. Torrado, E. Lopez, A. Centeno, et al., "Myocardin mRNA is augmented in the failing myocardium: expression profiling in the porcine model and human dilated cardiomyopathy," Journal of Molecular Medicine, vol. 81, no. 9, pp. 566-577, 2003. 
[26] M. O. Boluyt, L. O’Neill, A. L. Meredith, et al., "Alterations in cardiac gene expression during the transition from stable hypertrophy to heart failure: marked upregulation of genes encoding extracellular matrix components," Circulation Research, vol. 75, no. 1, pp. 23-32, 1994.

[27] M. Zungu, M. E. Young, W. C. Stanley, and M. F. Essop, "Expression of mitochondrial regulatory genes parallels respiratory capacity and contractile function in a rat model of hypoxia-induced right ventricular hypertrophy," Molecular and Cellular Biochemistry, vol. 318, no. 1-2, pp. 175-181, 2008.

[28] R. G. Kelly, M. Lemonnier, S. Zaffran, A. Munk, and M. E. Buckingham, "Cell history determines the maintenance of transcriptional differences between left and right ventricular cardiomyocytes in the developing mouse heart," Journal of Cell Science, vol. 116, no. 24, pp. 5005-5013, 2003.

[29] B. G. Bruneau, Z.-Z. Bao, D. Fatkin, et al., "Cardiomyopathy in Irx4-deficient mice is preceded by abnormal ventricular gene expression," Molecular and Cellular Biology, vol. 21, no. 5, pp. 1730-1736, 2001.

[30] H. Akazawa and I. Komuro, "Roles of cardiac transcription factors in cardiac hypertrophy," Circulation Research, vol. 92, no. 10, pp. 1079-1088, 2003.

[31] P. A. Modesti, S. Vanni, I. Bertolozzi, et al., "Different growth factor activation in the right and left ventricles in experimental volume overload," Hypertension, vol. 43, no. 1, pp. 101-108, 2004.

[32] J. Gallego-Delgado, S. B. Connolly, A. Lazaro, et al., "Transcriptome of hypertension-induced left ventricular hypertrophy and its regression by antihypertensive therapies," Hypertension Research, vol. 32, no. 5, pp. 347-357, 2009.

[33] T. Urashima, M. Zhao, R. Wagner, et al., "Molecular and physiological characterization of RV remodeling in a murine model of pulmonary stenosis," American Journal of PhysiologyHeart and Circulatory Physiology, vol. 295, no. 3, pp. H1351H1368, 2008. 

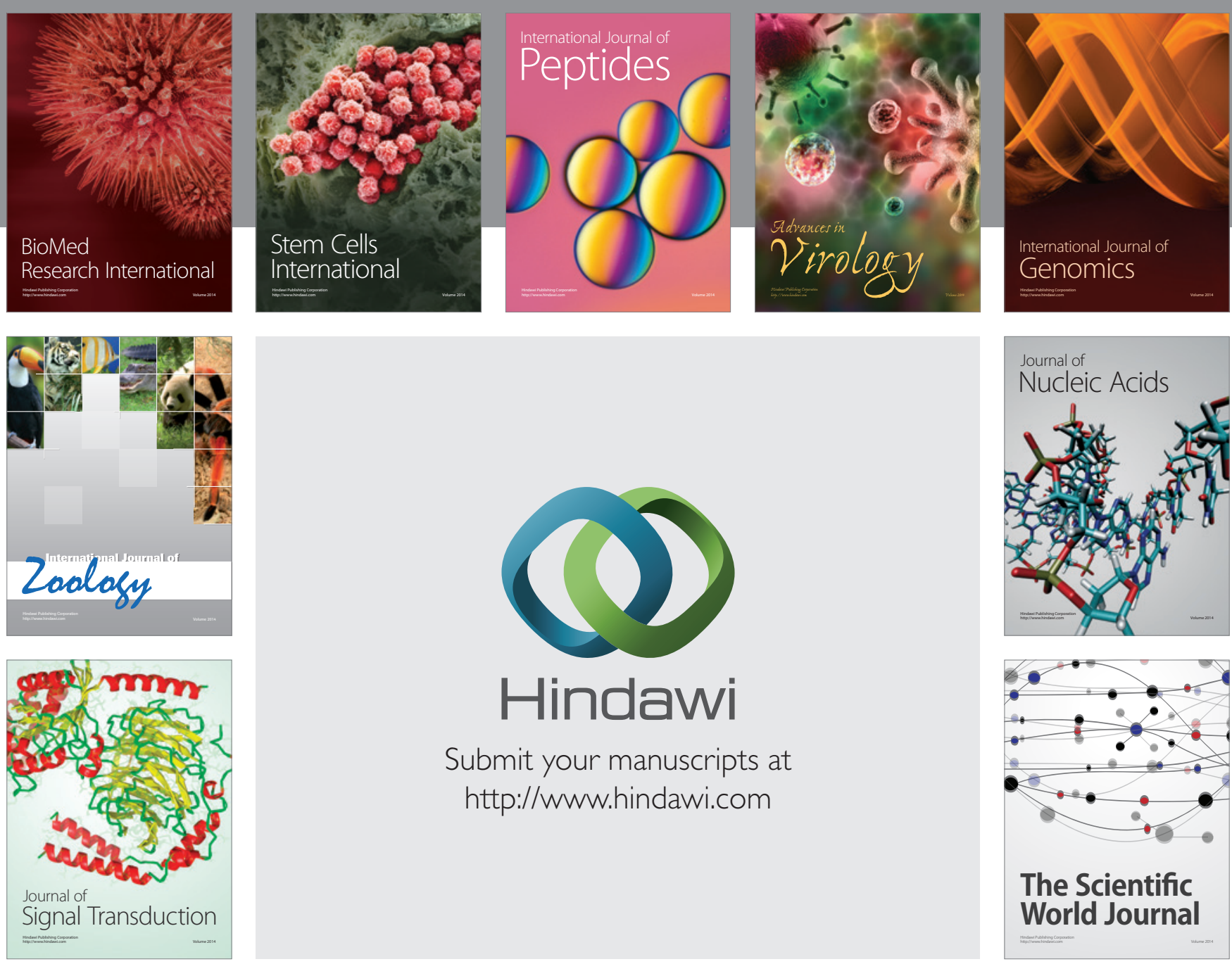

Submit your manuscripts at

http://www.hindawi.com
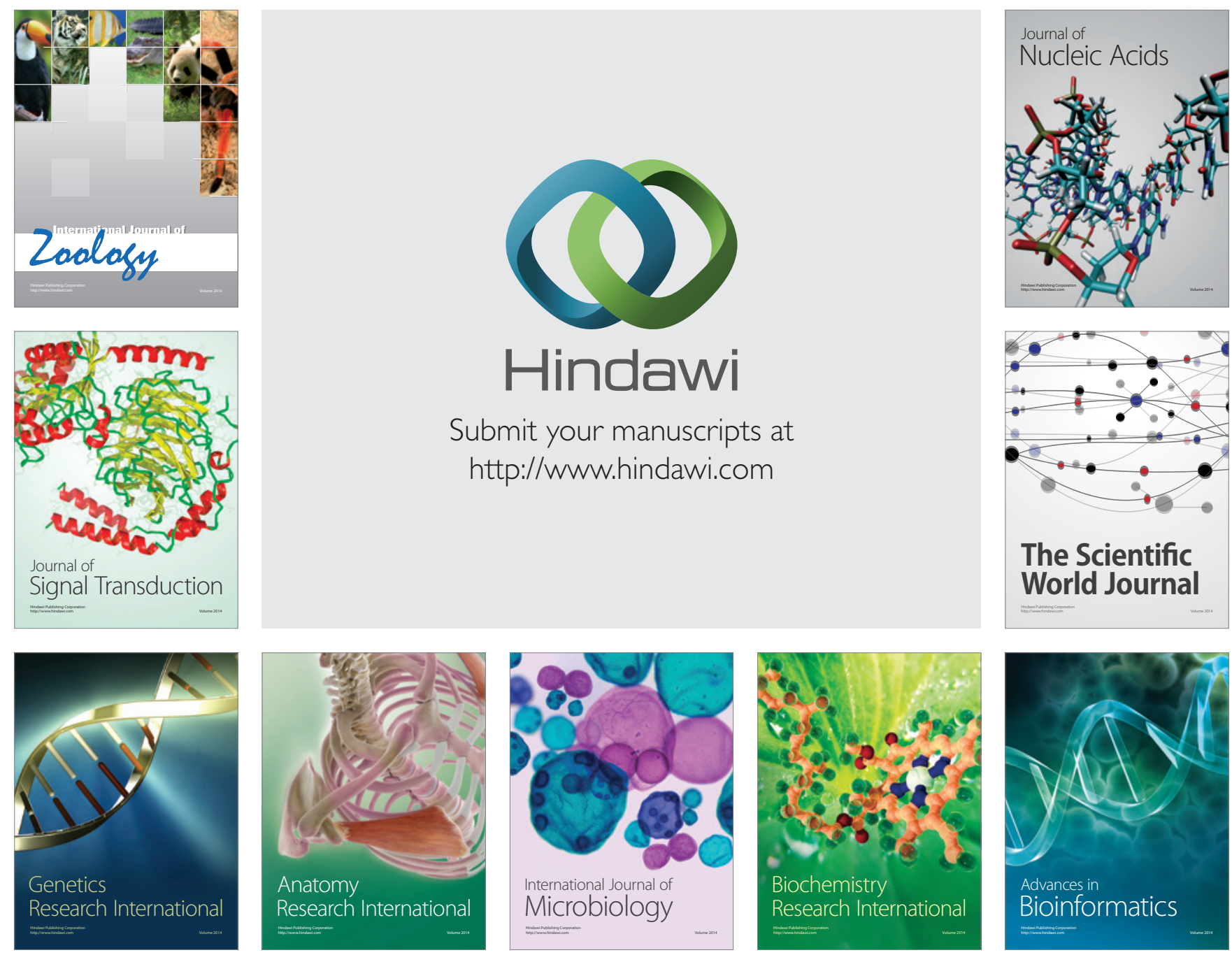

The Scientific World Journal
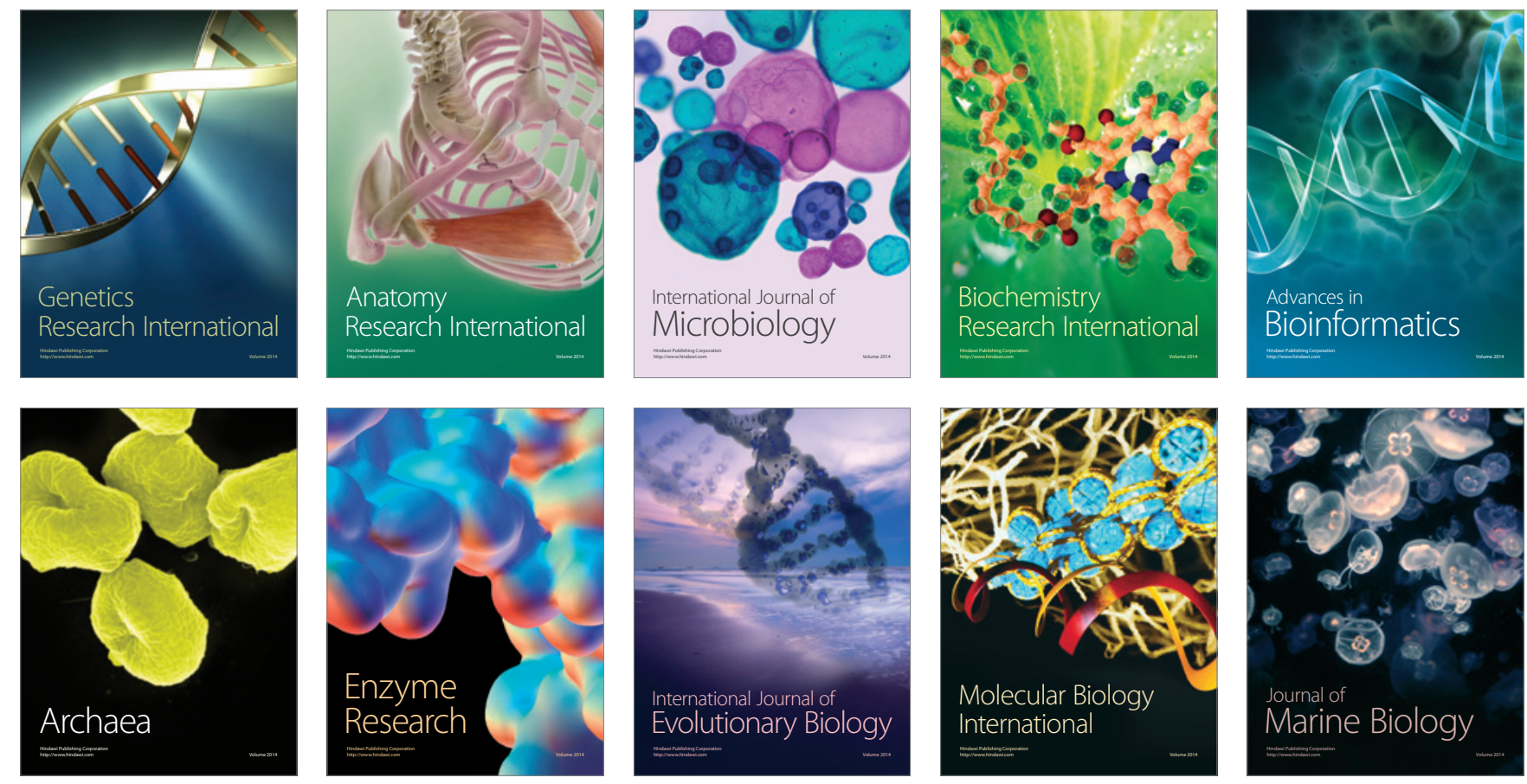